\title{
Cue-reactivity in behavioral addictions: A meta-analysis and methodological considerations
}

\author{
KATRIN STARCKE ${ }^{1,2}$, STEPHANIE ANTONS ${ }^{1,2}$, PATRICK TROTZKE $^{1,2}$ and MATTHIAS BRAND ${ }^{1,2 *}$ \\ ${ }^{1}$ Department of General Psychology: Cognition and Center for Behavioral Addiction Research (CeBAR), \\ University of Duisburg-Essen, Duisburg, Germany \\ ${ }^{2}$ Erwin L. Hahn Institute for Magnetic Resonance Imaging, Essen, Germany
}

(Received: December 21, 2017; revised manuscript received: April 6, 2018; accepted: April 7, 2018)

\begin{abstract}
Background and aims: Recent research has applied cue-reactivity paradigms to behavioral addictions. The aim of the current meta-analysis is to systematically analyze the effects of learning-based cue-reactivity in behavioral addictions. Methods: The current meta-analysis includes 18 studies (29 data sets, 510 participants) that have used a cue-reactivity paradigm in persons with gambling (eight studies), gaming (nine studies), or buying (one study) disorders. We compared subjective, peripheral physiological, electroencephal, and neural responses toward addiction-relevant cues in patients versus control participants and toward addiction-relevant cues versus control cues in patients. Results: Persons with behavioral addictions showed higher cue-reactivity toward addiction-relevant cues compared with control participants: subjective cue-reactivity $(d=0.84, p=.01)$ and peripheral physiological and electroencephal measures of cue-reactivity $(d=0.61, p<.01)$. Increased neural activation was found in the caudate nucleus, inferior frontal gyrus, median cingulate cortex, subgenual cingulate, and precentral gyrus. Persons with gambling, gaming, or buying disorders also showed higher cue-reactivity toward addiction-relevant cues compared with control cues: subjective cue-reactivity $(d=0.39, p=.11)$ and peripheral physiological and electroencephal measures of cue-reactivity $(d=0.47, p=.05)$. Increased neural activation was found in the caudate nucleus, inferior frontal gyrus, angular gyrus, inferior network, and precuneus. Discussion and conclusions: Cue-reactivity not only exists in substance-use disorders but also in gambling, gaming, and buying disorders. Future research should differentiate between cue-reactivity in addictive behaviors and cue-reactivity in functional excessive behaviors such as passions, hobbies, or professions.
\end{abstract}

Keywords: cue-reactivity, craving, behavioral addiction, gambling, gaming, buying

\section{INTRODUCTION}

Persons who are addicted to substances frequently show specific responses when they are confronted with substancerelevant cues. Responses include subjective, peripheral physiological, and neural responses. Carter and Tiffany (1999) published a meta-analysis including persons addicted to alcohol, nicotine, cocaine, or heroin. Subjective and peripheral physiological responses, such as heart rate, sweat gland activity, and skin temperature of the addicted persons, were analyzed. These reactions were compared between addiction-relevant cues versus control cues. Results indicate that addiction-relevant cues elicited cue-reactivity on a subjective, and also (albeit to a smaller degree) on peripheral physiological levels. Subjective arousal, heart rate, and sweat gland activity increase, whereas skin temperature decreases when addicted individuals are confronted with addiction-relevant cues. Since then, numerous studies have applied cue-reactivity paradigms in functional imaging studies and assessed brain activity during cue exposure (e.g., Chase, Eickhoff, Laird, \& Hogarth, 2011; Jasinska, Stein, Kaiser, Naumer, \& Yalachkov, 2014; Wilson, Sayette, \& Fiez, 2004). The results indicate that the prefrontal cortex, the anterior cingulate cortex, the ventral striatum, the insula, the hippocampus, and the amygdala are involved in cue-reactivity toward substance-related cues. Overall, cue-reactivity paradigms are established research tools for investigating substance-use disorders.

The basis of cue-reactivity is learning mechanisms, in which various cues become associated with the rewarding properties of the drug (Hyman, Malenka, \& Nestler, 2006; Tiffany \& Wray, 2011). These conditioned cues trigger conditioned emotional/motivational reactions (i.e., cuereactivity), which provide the basis for experiencing craving and comprise the anticipation of reward or the occurrence of withdrawal symptoms in the case of not consuming the substance. On a neural level, the dopaminergic mesolimbic reward system (especially the ventral striatum) plays a crucial role and Robinson and Berridge $(1993,2008)$ postulate within their incentive sensitization theory of addiction

* Corresponding author: Matthias Brand; Department of General Psychology: Cognition and Center for Behavioral Addiction Research (CeBAR), University of Duisburg-Essen, Forsthausweg 2, Duisburg 47057, Germany; Phone: +49203 3792541; Fax: +49 203 3791846; E-mail: matthias.brand@uni-due.de

This is an open-access article distributed under the terms of the Creative Commons Attribution-NonCommercial 4.0 International License, which permits unrestricted use, distribution, and reproduction in any medium for non-commercial purposes, provided the original author and source are credited, a link to the CC License is provided, and changes - if any - are indicated. 
that due to sensitization of the system, cues become more salient and in turn trigger craving reactions. Consumption behavior leads to the desired consequences, which enhances the probability to consume again (operant conditioning) (Drummond, 2001). In the course of addiction, there is a movement from initial ventral striatum activity (representing the hedonic "liking") to the dorsal striatum (representing the more compulsive and habituated "wanting") (Everitt \& Robbins, 2005). Particularly, the neural responses to cues can predict the risk of relapse after detoxification (Wrase, Grüsser, \& Heinz, 2006).

The term "addiction" is not restricted to substances. Decades ago, some authors considered different excessive behaviors to be addictive too (von Gebsattel, 1954). To date, only gambling disorder is included as behavioral addiction in the current fifth version of the Diagnostic and Statistical Manual for Mental Disorders (DSM-5) and Internet gaming disorder is included as a research diagnosis (American Psychiatric Association, 2013). However, there is a current debate whether to consider other behaviors, such as Internetuse disorder or buying disorder as addictive conditions as well (Potenza, 2006). Potential similarities between substance-related and behavioral addictions are the urge to perform the behavior, development of tolerance, withdrawal symptoms, unsuccessful attempts to stop or reduce the behavior, and continuation of the behavior despite negative consequences (Grüsser, Poppelreuter, Heinz, Albrecht, \& Sass, 2007). This has also been observed for behaviors, such as buying, Internet gaming, and others (Potenza, 2014). To date, research concerning behaviors other than gambling is limited and therefore they are not included in the current official classification systems of mental disorders (Potenza, 2014). In addition, it has been proposed not to pathologize common behaviors (Kardefelt-Winther et al., 2017). However, in recent years, studies were conducted that addressed cue-reactivity in the context of behavioral addictions (e.g., Niu et al., 2016). Persons who perform certain behaviors pathologically are confronted with behavior-relevant cues and their reactions are monitored. For gambling disorder, numerous studies on cue-reactivity exist (e.g., Balodis, Lacadie, \& Potenza, 2012; Goudriaan, de Ruiter, van den Brink, Oosterlaan, \& Veltman, 2010; McGrath, Dorbeck, \& Barret, 2013). The results indicate that individuals with gambling disorder react in a similar way to gamblingrelevant cues as individuals with substance-use disorder react to substance-associated cues. More precisely, when confronted with relevant cues, individuals with gambling disorder show subjective reactions (Grüsser, Plönzke, \& Albrecht, 2005), increased peripheral physiological arousal (Sharpe, Tarrier, Schotte, \& Spence, 1995), and increased brain activity in the dorsolateral prefrontal cortex (Crockford, Goodyear, Edwards, Quickfall, \& El-Guebaly, 2005), the striatum (Meng et al., 2014), and the amygdala (Goudriaan et al., 2010). Thus, behavioral addictions might have similarities with substance-use disorders, although no psychotropic substance is consumed. In a recent metaanalysis, Noori, Cosa Linan, and Spanagel (2016) compared brain activation toward the respective cues for substances, gambling, food, and sex. They found overlapping neural circuits for substance-use disorders and behaviors, i.e., activations in the anterior cingulate gyrus, insula, caudate head, inferior frontal gyrus, middle frontal gyrus, and cerebellum.

The present meta-analysis aims to quantify cue-reactivity for behaviors that are potentially addictive, such as gambling, gaming, buying, Internet usage, exercise, and work. Hypersexual behavior and food addiction were not included because of methodological considerations (see "Methods" section). We hypothesize that people who suffer from those behaviors show higher reactions when they are confronted with behavior-relevant cues compared with healthy control participants. We also hypothesize that people who suffer from those behaviors show higher reactions when they are confronted with behavior-relevant cues compared with control cues.

\section{METHODS}

\section{Inclusion criteria}

The study procedures were carried out in accordance with the Declaration of Helsinki. We concentrated our literature search on the following behaviors that are considered as potentially addictive: gambling, gaming, Internet usage, buying, exercise, and work. We excluded all types of eating disorders for the following reasons. First, eating includes ingestion and is a natural reward, which is a main difference to the other behavioral addictions. Second, feeding and eating disorders are a separate diagnostic category in the DSM-5. Third, the studies concerning cue-reactivity in eating disorders often include different outcome measures than other studies, for example, the amount of actual consumption. We further excluded studies with potential Internet pornography-use disorder (e.g., Prause, Steele, Stalay, Sabatinelli, \& Hajcak, 2015; Voon et al., 2014), because sexuality is also a natural reward and high cuereactivity toward sexual cues is not restricted to persons with pathological sexual behavior. Furthermore, it has been discussed that pornographic pictures do not represent cues but direct rewards, because seeing them is directly rewarding (Gola, Wordecha, Marchewka, \& Sescousse, 2016). In addition, we only included studies that used a classical cuereactivity paradigm in which patients were exposed to visual or auditory cues, while their subjective, physiological, or neural reactions were measured. That means, we excluded studies in which neuropsychological tasks were applied that were modified with cues (e.g., go/no-go tasks including addiction-relevant cues) or in which the addiction-relevant behavior was actually performed (e.g., playing a video game or simulated gambling).

\section{Literature search}

The databases PubMed and PsycInfo were reviewed for suitable articles. The search was conducted using the search terms "gambling addiction," "pathological gambling," "gambling disorder," "gaming addiction," "pathological gaming," "gaming disorder," "buying disorder," "buying addiction," "pathological buying," "compulsive buying," "shopping addiction," "pathological shopping," "shopping disorder," "compulsive shopping," "Internet addiction," 
"pathological Internet use," "Internet-use disorder," "Internet gaming disorder," "Internet gambling disorder," "Internetshopping disorder," "Internet-communication disorder," "exercise addiction," "compulsive exercise," "work addiction," "compulsive working," "workaholism," "cue," "cue exposure," "stimuli," "reactivity," "craving," "conditioned withdrawal," "conditioned responses," and "urges" in PubMed. In PsycInfo, capacities concerning the number of search terms were limited and the search terms "gambling addiction," "gaming addiction," "buying addiction," "Internet addiction," "exercise addiction," "work addiction," "cue," "cue exposure," "stimuli," "reactivity," "craving," "conditioned withdrawal," "conditioned responses," and "urges" were used. A search according to Boolean logic was performed, that is, each search term reflecting the behavioral addictions (using the conjunction OR) and each search term reflecting cue-reactivity (using the conjunction OR) were combined using the conjunction AND. Each term was required to be present in the "Title" of the paper. The search period was from January 1995 to September 2017. Further criteria were English language and humans as participants.

The studies were then analyzed as to whether they used the appropriate design, which means that persons with problematic or pathological behavior were investigated with a cue-reactivity paradigm. Cue-reactivity must have been compared between behavior-relevant cues and control cues on a subjective level (e.g., ratings of arousal), peripheral physiological or electroencephal level (e.g., skin conductance responses, heart rate, and electroencephalography), or neural level (e.g., functional magnetic resonance imaging and positron emission tomography). In addition, the references of the selected articles were reviewed for further literature and the papers by relevant authors were screened. Furthermore, an additional judge who was blind to our previous selections screened the references of the selected articles again. We also searched for unpublished data using the ProQuest databases to search for dissertations or master theses (www.proquest.com) and posted a request to send unpublished data on Research Gate (https:/www.researchgate.net/ home). A total of 18 studies (29 data sets and 510 participants) fulfilled the aforementioned criteria and the relevant data were available and could be included in the metaanalytical calculations. The studies include gambling (eight studies), gaming (nine studies), and buying (one study) disorder. All the studies that were included have been published. The exact searching and selecting procedure is shown in Figure 1. Within the reference list, studies that are included in the meta-analytical calculations are marked with the symbol * (Blanchard, Wulfert, Freidenberg, \& Malta, 2000; Crockford et al., 2005; Goudriaan et al., 2010; Han, Hwang, \& Renshaw, 2011; Han, Kim, Lee, \& Renshaw, 2012; Ko et al., 2009, 2011, 2013; Liu et al., 2017; Lorenz et al., 2012; McGrath et al., 2013; Potenza et al., 2003; Sharpe et al., 1995; Sodano \& Wulfert, 2010; Sun et al., 2012; Thalemann, Wölfling, \& Grüsser, 2007; Trotzke, Starcke, Pedersen, \& Brand, 2014; Wölfling et al., 2011).

\section{Coding of variables}

The following variables were coded: sample size $(N)$, age, gender, mean, and standard deviation $(S D)$ of cue-reactivity toward behavior-relevant cues and control cues in patients and healthy participants. Few studies used a baseline correction when presenting cue-reactivity data, whereas others did not. We used the raw data of cue-reactivity measures when they were provided and baseline-corrected values only if the raw data were not available. If baseline-corrected data were used, this is indicated in the "Results" section. If more than one type of control cues were used, we used those cues that were more proximal to the addiction-relevant cues [e.g., exciting cues (such as a rollercoaster drive) instead of neutral cues (such as unexciting nature cues)]. Reactions were assessed on a subjective, peripheral physiological, or neural level.

\section{Effect size (ES) calculation and meta-analytical calculations}

$N$, means, and $S D$ s of cue-reactivity were used to calculate Cohen's $d$ as ES. For the between-design (addictionrelevant cues only; patients vs. control participants), Cohen's $d$ was calculated as (patients' mean cue-reactivity toward addiction-relevant cues-control participants' mean cuereactivity toward addiction-relevant cues)/pooled $S D$ addiction-relevant cues (DeCoster, 2004; Field \& Gillet, 2010). Thus, positive $d$ scores represent higher cue-reactivity toward the addiction-relevant cues in patients than in control participants. For the within-design (patients only; addictionrelevant cues vs. control cues), Cohen's $d$ was calculated as (mean cue-reactivity toward addiction-relevant cues-mean cue-reactivity toward control cues)/SD cue-reactivity toward control cues (Becker, 1988). Thus, positive $d$ scores represent higher cue-reactivity toward the addiction-relevant cues than toward the control cues. We included the correlation between reactions toward addiction-relevant cues and control cues in the meta-analytical calculations. As not many studies provided these values, we estimated the correlation coefficients from those studies from which they were available and applied them to the rest of the studies. This was done separately for subjective data (two data sets were available) and for peripheral-physiological data (two data sets were available). Random ES model (Hunter \& Schmidt, 2004) was used for significance testing, confidence intervals, and $\chi^{2}$ tests of homogeneity according to Hedges and Vevea (1998). Significant $\chi^{2}$ tests indicate heterogeneity of the results.

For the brain imaging data, a voxel-based meta-analytic approach was used, which is implemented in the software Anisotropic ES-signed differential mapping (ES-SDM), version 5.141 (Radua \& Mataix-Cols, 2009; Radua et al., 2012). Two meta-analyses were calculated: (a) one for the comparison between patients and control participants [addicted (addiction-related cues vs. control cues) versus control participants (addiction-related cues vs. control cues)] and (b) other for the contrast addiction-relevant cues against control cues in patients (addiction-related cues vs. control cues). The more conservative contrast against control cues instead of the contrast against baseline was selected in studies, which reported multiple contrasts to avoid biasing meta-analysis results. In all included studies, the same threshold was used throughout the whole brain. $Z$ values from studies, which did not report $t$ values, were transformed using the ES-SDM 


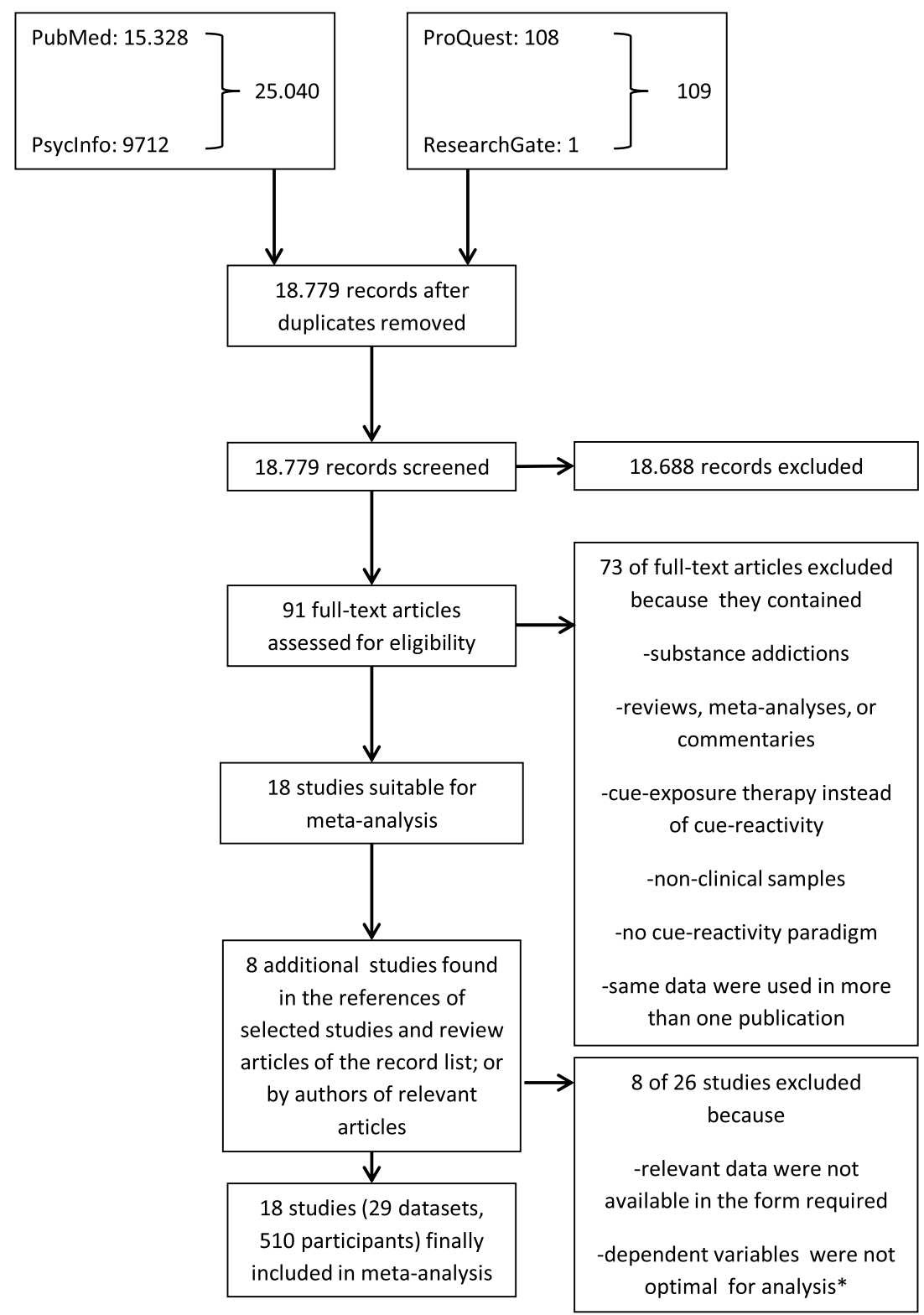

Figure 1. Flow chart of the literature search. ${ }^{*}$ This was the case in one study in which the only dependent variable was the subjective urge to perform the behavior. Subjective urges elicited by cues were excluded in the current analysis because the comparison between addictionrelevant cues and addiction-irrelevant cues is inappropriate in this case. Addiction-irrelevant cues should not elicit an urge to perform the addictive behavior and this would lead to an overestimation of effect size

web utilities (www.sdmproject.com/utilities). The detailed procedure of ES-SDM software has been described previously (Radua \& Mataix-Cols, 2009; Radua et al., 2012). The program converts the peak $t$ values to Hedges ES and uses those ESs and the peak coordinates to recreate maps of the ES of the BOLD response for the contrast of interest. This algorithm applies an anisotrophic non-normalized Gaussian kernel to the voxels close to the peak. Afterward, a voxelwise random effects meta-analysis is conducted, which weights the studies for sample size and variance. Default ES-SDM kernel size and thresholds were used as recommended by Radua et al. (2012) (FWHM =20 mm, $p<.005$, peak height $Z>1, k>10$ voxels). For purposes of visualization, results were superimposed on a single-subject Talairach template (colin27) (Kochunov et al., 2002), using the Multi-image Analysis GUI (version 3.8; Mango,
Research Imaging Institute, University of Texas Health Science Center at San Antonio, TX, USA).

\section{Publication bias}

As a first rough indicator of publication bias, the fail-safe $N$ procedure by Rosenthal (1979) was used. This procedure estimates the number of unpublished studies needed to turn a significant ES into a non-significant one. Then, Begg and Mazumdar's (1994) rank correlation test was applied. This test quantifies the relationship between ES and sample size, and a significant result indicates potential publication bias. Funnel plots as proposed, for example, by Vevea and Woods (2005) were not created because of the small number of data sets that could be included into each category (Higgins \& Green, 2011). 


\section{RESULTS}

The results of the meta-analytical calculations are presented according to the respective cue-reactivity measures. For subjective and peripheral physiological and electroencephal measures, a positive $d$ score means that patients react stronger toward addiction-relevant cues than control participants and that patients react stronger toward addiction-relevant cues than to control cues. In contrast to the meta-analysis by Carter and Tiffany (1999), studies on functional magnetic resonance imaging are also included here. Furthermore, there is a greater variety in peripheral physiological and electroencephal recordings. Therefore, we sorted our cue-reactivity measures according to the following three outcome measures: (a) subjective measures, (b) peripheral physiological and electroencephal measures, and (c) functional brain imaging data. After providing the pooled effects of gambling, gaming, and buying, we provide descriptive results for each of the addictions separately. We then report effects after excluding data on buying disorder, because only a single study on buying disorder was available. Finally, we report effects excluding data of the two studies that used baseline-corrected values instead of raw values.

\section{Characteristics of each study}

In Table 1, we provide the main characteristics of each study.

\section{Subjective cue-reactivity}

Variables that assessed how strong the specific cues elicited arousal, excitement, etc., were included, whereas variables that assessed how strong the cues elicited the urge to perform the behavior were excluded. Control cues are not suited to elicit an urge to perform a behavior. For example, the picture of a buffalo should not elicit the urge to buy the buffalo in individuals with buying disorder; the video of a landscape should not elicit the urge to gamble in individuals with gambling disorder. The inclusion of questions such as "how strong does the cue elicit the urge to perform a certain behavior?" would lead to overestimated effects. Furthermore, average scores were built in case that more than one subjective measure was assessed. If a questionnaire with different items, such as "pleasant," "stimulated," "satisfied," and "high," was presented after cue presentation, we averaged the scores of these items and only calculated a single ES $d$.

Addiction-relevant cues in patients versus control participants. For the six data sets available, an ES of $d=0.84$ was observed $(k=6$, lower $d=0.19$, upper $d=1.49$, standard error $=0.33, z=2.54, p=.01)$. The test for homogeneity was not significant $\left(\chi^{2}=3.80, d f=5\right.$, $p=.58)$. Rosenthal's fail-safe was $N=76$. Begg and Mazumdar's rank correlation test was not significant ( $\tau=0.47, p=.19)$. Out of the six data sets, four included data on gambling, and an ES of $d=0.45$ was observed; the ES of the single study on gaming was $d=1.00$; the ES of the single study on buying was $d=1.96$. Excluding data on buying disorder results in an ES of $d=0.52$ (remains significant). Excluding the two studies that used baseline-corrected values results in an ES of $d=1.07$ (remains significant).
Addiction-relevant cues versus control cues in patients. For the seven data sets available, an ES of $d=0.39$ was observed $(k=7$, lower $d=-0.09$, upper $d=0.86$, standard error $=0.24, z=1.59, p=.11)$. The test for homogeneity was not significant $\left(\chi^{2}=6.69, d f=6, p=.35\right)$. Rosenthal's fail-safe was $N=16$. Begg and Mazumdar's rank correlation test was not significant $(\tau=0.14, p=.65)$. Out of the seven data sets, five included data on gambling, and an ES of $d=0.09$ was observed; the ES of the single study on gaming was $d=1.74$; the ES of the single study on buying was $d=0.87$. Excluding data on buying disorder results in an ES of $d=0.29$ (remains insignificant). Excluding the two studies that used baseline-corrected values results in an ES of $d=0.61$ (reaches significance).

\section{Peripheral physiological and electroencephal cue-reactivity}

As peripheral physiological measures, heart rate, skin conductance level, skin conductance responses, skin resistance level, systolic blood pressure, and electromyography were used. In addition, electroencephalography was included.

Addiction-relevant cues in patients versus control participants. For the seven data sets available, an ES of $d=0.61$ was observed $(k=7$, lower $d=0.16$, upper $d=1.06$, standard error $=0.23, z=2.65, p<.01)$. The test for homogeneity was not significant $\left(\chi^{2}=6.12, d f=6, p=.41\right)$. Rosenthal's fail-safe was $N=51$. Begg and Mazumdar's rank correlation test was not significant $(\tau=0.42, p=.18)$. Out of the seven data sets, five included data on gambling (two studies assessed heart rate, one assessed skin conductance level, electromyography, and electroencephalography respectively), and an ES of $d=0.60$ was observed; the ES of the single study on gaming (electroencephalography) was $d=0.76$; the ES of the single study on buying (skin conductance responses) was $d=0.63$. Excluding data on buying disorder results in an ES of $d=0.62$ (remains significant). Excluding the two studies that used baseline-corrected values results in an ES of $d=0.92$ (remains significant).

Addiction-relevant cues versus control cues in patients. For the 11 data sets available, an ES of $d=0.47$ was observed $(k=11$, lower $d=0.00$, upper $d=0.94$, standard error $=$ $0.24, z=1.96, p=.05)$. The test for homogeneity was not significant $\left(\chi^{2}=10.11, d f=10, p=.43\right)$. Rosenthal's fail-safe was $N=59$. Begg and Mazumdar's rank correlation test was not significant $(\tau=-0.02, p=.94)$. Out of the 11 data sets, nine included data on gambling (four studies assessed heart rate, one assessed systolic blood pressure, skin resistance level, skin conductance level, electromyography, and electroencephalography, respectively), and an ES of $d=0.36$ was observed; the ES of the single study on gaming (electroencephalography) was $d=1.86$; the ES of the single study on buying (skin conductance responses) was $d=0.07$. Excluding data on buying disorder results in an ES of $d=0.51$ (changes from significant to marginally significant). Excluding the two studies that used baseline-corrected values results in an ES of $d=0.65$ (remains significant).

\section{Cue-reactivity in functional brain imaging studies}

All studies that were included used functional magnetic resonance imaging. All 3D coordinates of activation 
Table 1. Characteristics of the single studies

\begin{tabular}{|c|c|c|c|c|c|c|}
\hline $\begin{array}{l}\text { Addiction type and } \\
\text { study }\end{array}$ & $\begin{array}{c}\text { Number of } \\
\text { patients }\end{array}$ & $\begin{array}{l}\text { Characteristics of } \\
\text { patients }\end{array}$ & Stimulus type & $\begin{array}{l}\text { Preferred } \\
\text { stimuli }\end{array}$ & Stimulus order & $\begin{array}{l}\text { Cue- } \\
\text { reactivity } \\
\text { measures }\end{array}$ \\
\hline \multicolumn{7}{|l|}{ Gambling (eight studies) } \\
\hline Blanchard et al. (2000) & $7(0 \%$ f) & Mixed gamblers ${ }^{\mathrm{a}}$ & $\begin{array}{l}\text { Auditory and } \\
\text { mental } \\
\text { imagery }\end{array}$ & Yes & $\begin{array}{l}\text { Same for all } \\
\text { participants }\end{array}$ & $\begin{array}{l}\text { HR } \\
\text { SBP } \\
\text { SRL }\end{array}$ \\
\hline Crockford et al. (2005) & $10(0 \% \mathrm{f})$ & Mixed gamblers & Video & No & $\begin{array}{l}\text { Block design } \\
\text { counterbalanced }\end{array}$ & fMRI \\
\hline Goudriaan et al. (2010) & $17(0 \%$ f) & Mixed gamblers & Photographs & No & Randomized & fMRI \\
\hline McGrath et al. (2013) & $10(30.77 \% \mathrm{f})$ & $\begin{array}{l}\text { Video lottery } \\
\text { terminal gamblers }\end{array}$ & $\begin{array}{l}\text { Photographs } \\
\text { sounds }\end{array}$ & No & $\begin{array}{l}\text { Same for all } \\
\text { participants }\end{array}$ & $\begin{array}{c}\text { Subjective } \\
\text { HR }\end{array}$ \\
\hline Potenza et al. (2003) & $10(0 \% \mathrm{f})$ & Mixed gamblers & Videos & No & Counterbalanced & $\begin{array}{l}\text { Subjective } \\
\text { fMRI }\end{array}$ \\
\hline Sharpe et al. $(1995)^{b}$ & $13(38.46 \% \mathrm{f})$ & Mixed gamblers & Videos & No & Randomized & $\begin{array}{c}\text { SCL } \\
\text { HR } \\
\text { EMG } \\
\text { Subjective }\end{array}$ \\
\hline $\begin{array}{l}\text { Sodano and Wulfert } \\
(2010)^{\mathrm{b}}\end{array}$ & $21(28.57 \%$ f $)$ & Mixed gamblers & Videos & Yes & Counterbalanced & $\begin{array}{c}\text { HR } \\
\text { Subjective }\end{array}$ \\
\hline Wölfling et al. (2011) & $15(20.00 \% \mathrm{f})$ & Mixed gamblers & Photographs & No & $\begin{array}{l}\text { Pseudorandomized } \\
\text { block design }\end{array}$ & $\begin{array}{c}\text { EEG } \\
\text { Subjective }\end{array}$ \\
\hline \multicolumn{7}{|l|}{ Gaming (nine studies) } \\
\hline Han et al. (2011) & $11(0 \% \mathrm{f})$ & StarCraft gamers & Videos & Yes & Block design & fMRI \\
\hline Han et al. (2012) & 15 (n.a.) & Mixed gamers ${ }^{\mathrm{a}}$ & Videos & No & Block design & fMRI \\
\hline Ko et al. (2009) & $10(0 \% \mathrm{f})$ & $\begin{array}{l}\text { World of Warcraft } \\
\text { gamers }\end{array}$ & Pictures & Yes & Block design & fMRI \\
\hline Ko et al. (2011) & $15(0 \% \mathrm{f})$ & $\begin{array}{l}\text { Gamers of the same } \\
\text { online game }\end{array}$ & Pictures & Yes & Pseudorandom & fMRI \\
\hline Ko et al. (2013) & $16(0 \% \mathrm{f})$ & Gamers & Pictures & No & Pseudorandom & fMRI \\
\hline Liu et al. (2017) & $39(0 \%$ f) & Gamers & Pictures & Yes & Block design & fMRI \\
\hline Lorenz et al. (2012) & $8(0 \%$ f) & $\begin{array}{l}\text { World of Warcraft } \\
\text { gamers }\end{array}$ & Pictures & No & Block design & $\begin{array}{c}\text { fMRI } \\
\text { Subjective }\end{array}$ \\
\hline Thalemann et al. (2007) & 15 (0\% f) & Mixed gamers & Pictures & No & Pseudorandom & EEG \\
\hline Sun et al. (2012) & $10(0 \% \mathrm{f})$ & $\begin{array}{l}\text { World of Warcraft } \\
\text { gamers }\end{array}$ & Pictures & No & Block design & fMRI \\
\hline $\begin{array}{l}\text { Buying (one study) } \\
\text { Trotzke et al. (2014) }\end{array}$ & $30(73.33 \%$ f) & Mixed buyers ${ }^{\mathrm{a}}$ & Photographs & Yes & Randomized & $\begin{array}{l}\text { Subjective } \\
\text { SCR }\end{array}$ \\
\hline
\end{tabular}

Note. f: female; SCR: skin conductance responses; HR: heart rate; SBP: systolic blood pressure; SRL: skin resistance level; fMRI: functional magnetic resonance imaging; SCL: skin conductance level; EMG: electromyography; EEG: electroencephalography.

${ }^{a}$ Mixed gamblers, mixed gamers, and mixed buyers. Participants with different preferences were included (e.g., buying clothes, shoes, cosmetics, books, CDs, etc.). In some studies, preferred stimuli were used, i.e., only those stimuli that individually attract the participants (e.g., pictures of clothes for individuals with buying disorder who prefer buying clothes and no pictures of other products).

${ }^{\mathrm{b}}$ Results are baseline-corrected.

maxima were reported in standardized stereotactic space (Montreal Neurological Institute or Talairach).

Addiction-relevant cues in patients versus control participants. For the 10 data sets available, contrasting activations in patients and control participants, the analysis revealed six clusters, shown in Figure 2 and described in Table 2. In the right hemisphere, clusters were found in the median cingulate cortex (anterior cingulate, posterior cingulate, and paracingulate gyri), inferior frontal gyrus, and caudate nucleus. In the left hemisphere, activations were found in precentral gyrus and caudate nucleus. Moreover, activation in the subgenual cingulate was found. Out of the 10 data sets, two included data on gambling. With a threshold probability of $p<.01$, the analysis revealed significant activations in right striatum, right fusiform gyrus, median cingulate gyrus, middle occipital gyrus, and right inferior frontal gyrus. Eight studies included data on gaming. Significant clusters (with $p<.01$ ) were found in median cingulate, right inferior frontal gyrus, precentral gyrus, right caudate nucleus, left precentral gyrus, right superior temporal gyrus, corpus callosum, and right precuneus.

Addiction-relevant cues versus control cues in patients. For the six data sets available, the ES-SDM meta-analysis revealed six clusters of significant convergence across studies when contrasting addiction-relevant cues with 


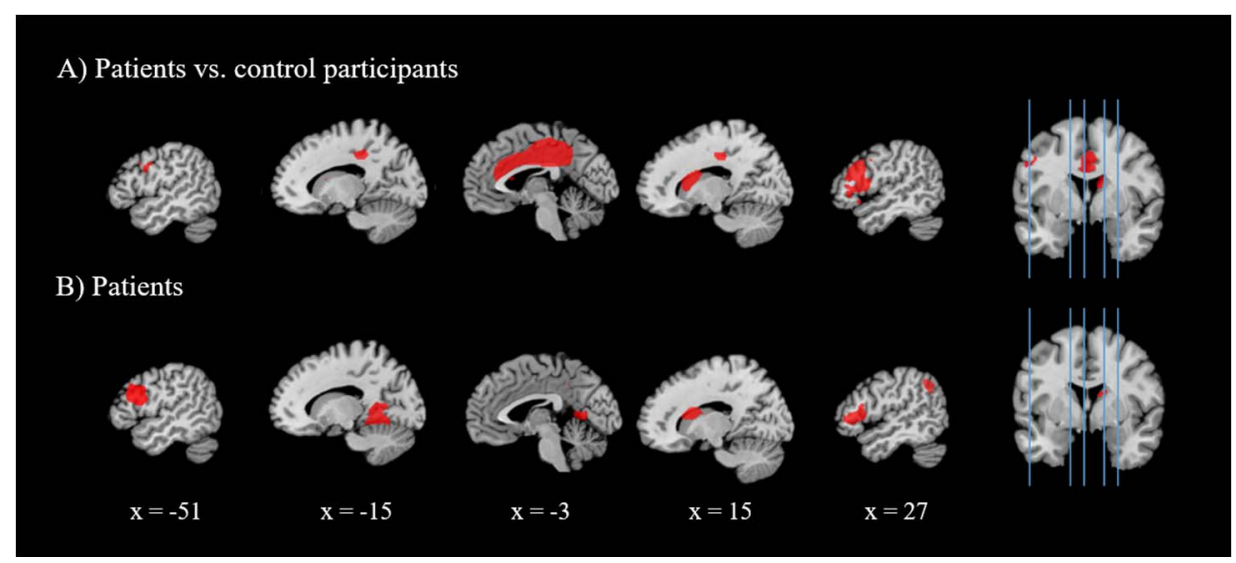

Figure 2. Brain activations in response to addiction-relevant cues: (A) in patients in contrast to control participants and (B) in patients in contrast to control cues. Blue lines display location of slices on the coronal axis

Table 2. Regions of activation in which patients showed higher activation to addiction-relevant cues than control participants

\begin{tabular}{|c|c|c|c|c|c|c|c|}
\hline & & \multirow[b]{2}{*}{ BA } & \multicolumn{3}{|c|}{ MNI } & \multirow[b]{2}{*}{ SDM-Z } & \multirow[b]{2}{*}{ Voxels } \\
\hline \multicolumn{2}{|c|}{ Anatomical region } & & $x$ & $y$ & $z$ & & \\
\hline $\mathrm{R}$ & Median cingulate & 23 & 0 & -16 & 30 & 3.678 & 3,956 \\
\hline $\mathrm{R}$ & Inferior frontal gyrus & 48 & 48 & 24 & 26 & 2.912 & 1,383 \\
\hline $\mathrm{R}$ & Caudate nucleus & & 16 & 10 & 8 & 2.870 & 374 \\
\hline $\mathrm{L}$ & Precentral gyrus & & -50 & 4 & 28 & 2.350 & 105 \\
\hline \multirow[t]{2}{*}{$\mathrm{L}$} & Caudate nucleus & & -10 & 10 & 16 & 2.192 & 30 \\
\hline & Corpus callosum & & 0 & 12 & 14 & 2.820 & 21 \\
\hline
\end{tabular}

Note. MNI: Montreal Neurological Institute; L: left; R: right; BA: broca area; SDM: signed differential mapping.

Table 3. Regions of activation in which addiction-relevant cues elicited greater activation than control cues in the patient group

\begin{tabular}{|c|c|c|c|c|c|c|c|}
\hline & & \multirow[b]{2}{*}{$\mathrm{BA}$} & \multicolumn{3}{|c|}{ MNI } & \multirow[b]{2}{*}{ SDM-Z } & \multirow[b]{2}{*}{ Voxels } \\
\hline \multicolumn{2}{|c|}{ Anatomical region } & & $x$ & $y$ & $z$ & & \\
\hline $\mathrm{L}$ & Inferior network & & -22 & -56 & -4 & 2.678 & 110 \\
\hline $\mathrm{L}$ & Inferior frontal gyrus & 48 & -46 & 28 & 20 & 2.282 & 661 \\
\hline $\mathrm{R}$ & Inferior frontal gyrus & 47 & 50 & 30 & -4 & 2.285 & 442 \\
\hline $\mathrm{R}$ & Caudate nucleus & & 10 & 8 & 10 & 2.260 & 244 \\
\hline $\mathrm{R}$ & Angular gyrus & 39 & 50 & -60 & 38 & 2.460 & 187 \\
\hline $\mathrm{R}$ & Precuneus & & 6 & -48 & 40 & 2.132 & 38 \\
\hline
\end{tabular}

Note. MNI: Montreal Neurological Institute; L: left; R: right; BA: broca area; SDM: signed differential mapping.

control cues, which are shown in Figure 2 and described in Table 3. In the right hemisphere, a cluster with activations in the inferior frontal gyrus, caudate nucleus, angular gyrus, and precuneus were found and in the left hemisphere, a cluster with activations in inferior network (inferior longitudina fasciculus, calcarine fissure, and fusiform gyrus) and inferior frontal gyrus were found. Out of the six data sets, one included data on gambling. In this study (Crockford et al., 2005), significant activations were found in bilateral precuneus, inferior parietal lobule, occipital cortex, cuneus, lingual gyrus, fusiform gyrus, right inferior frontal gyrus, and right parahippocampal gyrus. Five studies included data on gaming. The SDM meta-analysis revealed significant clusters (with $p<.01$ ) in bilateral inferior frontal gyrus, corpus callosum, right caudate nucleus, right angular gyrus, and bilateral cingulate gyrus.

\section{DISCUSSION AND CONCLUSIONS}

\section{Summary of evidence and interpretation}

Individuals with gambling, gaming, and buying disorders show higher cue-reactivity toward addiction-relevant cues compared with healthy control participants. Pooled data for subjective ratings of cue-reactivity reached significance. The ES was large. Pooled data for peripheral physiological and electroencephal measures also reached significance. The ES was medium. On a neural level, significant activations were observed in the caudate nucleus, the median cingulate cortex, the subgenual cingulate, the inferior frontal gyrus, and the precentral gyrus. Individuals with gambling, gaming, and buying disorders also show higher cue-reactivity toward addiction-relevant cues compared with control cues 
except for subjective ratings. Pooled data for subjective ratings of cue-reactivity did not reach significance. The ES was only small to medium. Pooled data for peripheral physiological and electroencephal measures reached significance. The ES was medium. On the brain level, significant activations were observed in the caudate nucleus, inferior frontal gyrus, angular gyrus, precuneus, and inferior network. Overall, the results indicate that cue-reactivity is not only restricted to substance-use disorders but also occurs in behavioral addictions. The only non-significant finding was the subjective rating of addiction-relevant cues compared with control cues within the patient group. The results are overall - in line with meta-analyses on cue-reactivity in substance-use disorders (Carter \& Tiffany, 1999; Noori et al., 2016).

It has been proposed that similar mechanisms underlie the development and maintenance of substance-use disorder and behavioral addictions. As outlined in the introduction, conditioning processes, such as classical and operant conditioning, can lead to desires of substances as well as behaviors (Grüsser et al., 2007). Relevant cues are associated with the consequences of a behavior (by classical conditioning) and elicit a motivational state (cuereactivity) that leads to the desire to perform the behavior (craving). The actual performance of the behavior leads to the desired consequences, which enhances the probability to perform it again (operant conditioning) (Drummond, 2001). The results of the functional imaging studies indicate increased activation of regions that are associated with the anticipation and processing of rewards, and reward-related decision-making, such as the caudate nucleus and the median cingulate (Liu, Hairston, Schrier, \& Fan, 2011). The caudate nucleus is part of the dorsal striatum and this region is associated with cue-reactivity processing in chronic-addicted individuals who show habituated, compulsive consumption patterns. It is well described in addiction research that there is a movement from initial ventral striatum activity to the dorsal striatum in the course of addictions (Everitt \& Robbins, 2005). Not all behavioral addictions are officially classified and therapeutic interventions are limited, several patients may suffer from chronic addiction. Furthermore, the inferior frontal gyrus is involved in the detection of important cues (Hampshire, Chamberlain, Monti, Duncan, \& Owen, 2010). Current results emphasize that the increased salience for addiction-relevant cues, which was first established in the studies of substance-use disorder, can also be assumed for behavioral addictions.

Recently, gambling disorder has been moved from impulse control disorders to the category of substancerelated and addictive disorders in the DSM-5 and Internet gaming disorder has been listed as condition that needs further research and potentially belongs to this new category. Other behaviors, such as buying disorder, have not been classified to date, because the empirical evidence concerning these behaviors is still limited (Potenza, 2014). However, meanwhile, some new studies have been published and cue-reactivity paradigms have been established in persons with problematic behaviors other than gambling. In the current meta-analysis, ESs that were observed in patients with gaming and buying disorders were even higher than those that were observed in patients with gambling disorder in most of the analyses. This is a descriptive finding, but it is important to note that cuereactivity in gaming and buying disorders might even exceed cue-reactivity in the only disorder that is officially classified as a behavioral addiction in the DSM-5. On the brain level, similar regions are involved in cue-reactivity in patients with gambling disorder and those with gaming disorder, namely right inferior frontal gyrus, striatum (caudate nucleus), and cingulate cortex. The results indicate that similar mechanisms may be involved in the development and maintenance of gambling, gaming, and buying disorders. Consequently, gaming and buying disorders should also be considered as candidates for the category substance-related and addictive disorders in future versions of the DSM and the ICD.

Several researchers emphasize that common behaviors should not be pathologized and that not all excessive behaviors are addictive in nature (Kardefelt-Winther et al., 2017). For example, behavior, that is potentially harmful but results from a willful choice, should not be regarded as addictive. Future work should attempt to differentiate cue-reactivity in persons who are addicted to a behavior from those who are engaged in a certain behavior as the results of a willful choice.

\section{Limitations}

A relevant limitation of the meta-analysis is that the overall number of studies is small. The possibility exists that there are studies that were not covered by any of our database searches or that are published nowhere. Furthermore, in some studies, relevant data were not provided in the form required, which also led to a reduction of data available. We did not find appropriate studies for all potential behavioral addictions we intended to include. Thus, we did not find any studies that used cue-reactivity paradigms in clinical samples with potential further behavioral addictions, such as exercise addiction or workaholism. In addition, the number of studies that investigated cue-reactivity in each category was small. Therefore, potential differences between gambling, gaming, and buying disorders were only reported on a descriptive level and on the brain level with an increased threshold probability of $p<.01$. Only one of the studies reported data on buying disorder. Therefore, we performed a sensitivity analysis using the leave-one-out method and excluded data from this study. The results basically remained stable.

Many of the studies included contain more than one data set (e.g., subjective and physiological data), which means that the overall number of data sets is larger than the number of studies. Including multiple data sets of the same study has also been regarded as a problem. However, Ishak, Platt, Joseph, and Hanley (2008) assumed that dependencies do not change the results and so we did not change the common meta-analytical calculation methods. The Begg and Mazumdar's (1994) rank correlation test did not indicate publication bias. However, in small meta-analyses, a nonsignificant result does not guarantee the absence of publication biases. Funnel plots as proposed, for example, by Vevea and Woods (2005) are principally suited for small meta-analyses, but at least 10 data sets should be included 
in each calculation (Higgins \& Green, 2011), which was not the case for all calculations.

Another major limitation of the current meta-analysis is the heterogeneity of studies. Some studies were conducted with pictures as cues, others used videos or audiotapes. The dependent variables also varied among studies. For example, the subjective cue ratings represented experiences of arousal elicited by the cue, liking the cue, or the urge to perform the behavior when exposed to the cue. Variables that assessed the urge to perform the behavior had to be excluded from the analysis, because control cues were unsuited for this assessment. Therefore, no conclusions about subjective urges can be drawn here. Furthermore, subjective ratings were frequently assessed by questionnaires, which contained more than one question and were averaged for the analysis. Therefore, the overall value for subjective responses is unspecific. The same limitation occurred for the physiological data, which were combined and included various outcome measures (skin conductance, heart rate, blood pressure, electromyography, and electroencephalography). The control stimuli also differed between studies, i.e., some used neutral cues as control cues, whereas others used emotional addiction-irrelevant cues as control cues. Another shortcoming is that the studies used different contrasting procedures. While for the majority of studies, the raw data were used for the meta-analysis, two of the studies only reported results that were baseline-corrected. Including them led to decreased effects, so our calculations were conservative in this regard. Furthermore, most studies contrasted patients' cue-reactivity to addiction-relevant cues versus control cues and also contrasted patients' cuereactivity to addiction-relevant cues with a healthy control group. However, some studies only reported either of the above category. The studies also differed concerning the order of stimulus presentation (e.g., same for all participants vs. randomized). Concerning the participants, the proportion of female participants was low except for individuals with buying disorder. Furthermore, the inclusion of participants differed, i.e., while some studies investigated only patients with the same specific problematic behavior (e.g., who play a specific online game) and exposed them to their preferred cues, other studies investigated patients with mixed behaviors (e.g., who play any online game). Accordingly, some studies used cues that strongly resembled the patients' preferred cues, whereas in other studies, cues were less specific. A limitation that directly results from the fact that the behavioral addictions (besides gambling disorder) are not included in the official diagnostic systems is that not all patients had an official diagnosis. We included only studies in which the participants showed at least problematic behavior, but the threshold for defining a problematic behavior may have varied among the studies.

\section{Implications for future research}

More knowledge concerning cue-reactivity in behavioral addictions would allow the application of neuropsychological tasks, which are modified with behavior-relevant cues. In patient groups with behavioral addictions, relevant cues should influence cognitive processes (Brand, Young, Laier, Wölfling, \& Potenza, 2016). The assumption that cue-reactivity triggers craving reactions, which can lead to poor executive functions (such as reduced inhibitory processes) and finally lead to poor decision-making (performing the behavior, which is short-term rewarding, but has negative long-term consequences), could be tested in pathologies other than gambling and gaming. Furthermore, it is rational to study decision-making processes directly when confronted with addiction-relevant cues. Some recent studies in this field exist for buying (Raab, Elger, Neuner, \& Weber, 2011) and gambling disorders (e.g., Miedl, Büchel, \& Peters, 2014).

Importantly, research on cue-reactivity in behavioral addictions could contribute to the debate whether behavioral addictions other than gambling exist and what constitutes a behavioral addiction. To avoid an inflationary use of the term "behavioral addiction," it is essential to differentiate them from behaviors that are executed willfully, such as hobbies or professions (Kardefelt-Winther et al., 2017). Therefore, it would be useful to compare reactions to cues between persons whose excessive behavior is part of a willful choice (e.g., professional poker players and professional athletes) and persons whose excessive behavior cannot be explained by a willful choice (e.g., patients with gambling disorder who lose all their money and neglect their jobs and families and patients with running addiction who neglect their jobs, families, and health). The studies would profit from the assessment of cue-reactivity combining physiological and neural responses with fine-grained subjective measures. Subjective measures should be able to differentiate the hedonic "liking," and the more compulsive "wanting" component toward cues, which could be related to the specific physiological or neural responses. Such research could contribute to the debate on whether each hedonic behavior can become addictive and what discriminates excessive (but functional) from addictive (dysfunctional) behavior.

Funding sources: This work was funded in part by the German Research Foundation (grant number: STA 1196/2-3).

Authors' contribution: KS designed the study, screened the literature, analyzed the data, and wrote the manuscript. SA screened the literature and analyzed the data. PT and MB designed the study and revised the manuscript.

Conflict of interest: The authors declare no conflict of interest.

Acknowledgements: The authors would like to thank Lena Kölmel for her valuable help with the literature screening.

\section{REFERENCES}

References marked with an asterisk were included in the metaanalytical calculations.

American Psychiatric Association. (2013). Diagnostic and statistical manual for mental disorders (5th ed.). Arlington, VA: American Psychiatric Association. 
Balodis, I. M., Lacadie, C. M., \& Potenza, M. N. (2012). A preliminary study of the neural correlates of the intensities of self-reported gambling urges and emotions in men with pathological gambling. Journal of Gambling Studies, 28(3), 493-513. doi:10.1007/s10899-011-9259-8

Becker, B. J. (1988). Synthesizing standardized mean-change measures. British Journal of Mathematical and Statistical Psychology, 41(2), 257-278. doi:10.1111/j.2044-8317.1988. tb00901.x

Begg, C. B., \& Mazumdar, M. (1994). Operating characteristics of a rank correlation test for publication bias. Biometrics, 50(4), 1088-1101. doi: $10.2307 / 2533446$

*Blanchard, E. B., Wulfert, E., Freidenberg, B. M., \& Malta, L. S. (2000). Psychophysiological assessment of compulsive gamblers' arousal to gambling cues: A pilot study. Applied Psychophysiology and Biofeedback, 25(3), 155-165. doi:10. 1023/A:1009550724836

Brand, M., Young, K. S., Laier, C., Wölfling, K., \& Potenza, M. N. (2016). Integrating psychological and neurobiological considerations regarding the development and maintenance of specific Internet-use disorders: An interaction of PersonAffect-Cognition-Execution (I-PACE) model. Neuroscience and Biobehavioral Reviews, 71, 252-266. doi:10.1016/j. neubiorev.2016.08.033

Carter, B. L., \& Tiffany, S. T. (1999). Meta-analysis of cuereactivity in addiction research. Addiction, 94(3), 327-340. doi:10.1046/j.1360-0443.1999.9433273.x

Chase, H. W., Eickhoff, S. B., Laird, A. R., \& Hogarth, L. (2011). The neural basis of drug stimulus processing and craving: An activation likelihood estimation meta-analysis. Biological Psychiatry, 70(8), 785-793. doi:10.1016/j.biopsych. 2011.05.025

*Crockford, D. N., Goodyear, B., Edwards, J., Quickfall, J., \& El-Guebaly, N. (2005). Cue-induced brain activity in pathological gamblers. Biological Psychiatry, 58(10), 787-795. doi:10.1016/j.biopsych.2005.04.037

DeCoster, J. (2004). Meta-analysis notes. Retrieved January, 09, 2015, from www.stat-help.com/notes.html

Drummond, D. C. (2001). Theories of drug craving, ancient and modern. Addiction, 96(1), 33-46. doi:10.1046/j.1360-0443. 2001.961333.x

Everitt, B. J., \& Robbins, T. W. (2005). Neural systems of reinforcement for drug addiction: From actions to habits to compulsions. Nature Neuroscience, 8(11), 1481-1489. doi:10. 1038/nn1579

Field, A. P., \& Gillet, R. (2010). How to do a meta-analysis. British Journal of Mathematical and Statistical Psychology, 63(3), 665-694. doi:10.1348/000711010X502733

Gola, M., Wordecha, M., Marchewka, A., \& Sescousse, G. (2016). Visual sexual stimuli-cue or reward? A perspective for interpreting brain imaging findings in human sexual behaviors. Frontiers in Human Neuroscience, 10, 402, eCollection 2016. doi:10.3389/fnhum.2016.00402

*Goudriaan, A. E., de Ruiter, M. B., van den Brink, W., Oosterlaan, J., \& Veltman, D. J. (2010). Brain activation patterns associated with cue reactivity and craving in abstinent problem gamblers, heavy smokers and healthy controls: An fMRI study. Addiction Biology, 15(4), 491-503. doi:10.1111/j.1369-1600.2010.00242.x

Grüsser, S. M., Plönzke, B., \& Albrecht, U. (2005). Pathological gambling: An empirical study of the desire for addictive substances. Der Nervenarzt, 76(5), 592-596. doi:10.1007/ s00115-004-1764-x

Grüsser, S. M., Poppelreuter, S., Heinz, A., Albrecht, U., \& Sass, H. (2007). Verhaltenssucht. Eine eigenständige diagnostische Einheit? [Behavioural addiction. An independent diagnostic category?]. Der Nervenarzt, 78(9), 997-1002. doi:10.1007/ s00115-007-2281-5

Hampshire, A., Chamberlain, S. R., Monti, M. M., Duncan, J., \& Owen, A. M. (2010). The role of the right inferior frontal gyrus: Inhibition and attentional control. Neuroimage, 50(3), 13131319. doi:10.1016/j.neuroimage.2009.12.109

*Han, D. H., Hwang, J. W., \& Renshaw, P. F. (2011). Bupropion sustained release treatment decreases craving for video games and cue-induced brain activity in patients with Internet video game addiction. Psychology of Popular Media Culture, 1, 108-117. doi:10.1037/2160-4134.1.S.108

*Han, D. H., Kim, S. M., Lee, Y. S., \& Renshaw, P. F. (2012). The effect of family therapy on the changes in the severity of on-line game play and brain activity in adolescents with on-line game addiction. Psychiatry Research: Neuroimaging, 202(2), 126-131. doi:10.1016/j.pscychresns.2012.02.011

Hedges, L. V., \& Vevea, J. L. (1998). Fixed- and randomeffects models in meta-analysis. Psychological Methods, 3(4), 486-504. doi:10.1037/1082-989X.3.4.486

Higgins, J. P. T., \& Green, S. (2011, March). Cochrane handbook for systematic reviews of interventions version 5.1.0. Retrieved December 9, 2015, from www.cochrane-handbook.org

Hunter, J. E., \& Schmidt, F. L. (2004). Methods of meta-analysis: Correcting error and bias in research findings (2nd ed.). Newbury Park, CA: Sage.

Hyman, S. E., Malenka, R. C., \& Nestler, E. J. (2006). Neural mechanisms of addiction: The role of reward-related learning and memory. Annual Review of Neuroscience, 29(1), 565-598. doi:10.1146/annurev.neuro.29.051605.113009

Ishak, K., Platt, R. W., Joseph, L., \& Hanley, J. A. (2008). Impact of approximating or ignoring within-study covariances in multivariate meta-analyses. Statistics in Medicine, 27(5), 670-686. doi:10.1002/sim.2913

Jasinska, A., Stein, E. A., Kaiser, J., Naumer, M. J., \& Yalachkov, Y. (2014). Factors modulating neural reactivity to drug cues in addiction: A survey of human neuroimaging studies. Neuroscience and Biobehavioral Reviews, 38, 1-16. doi:10.1016/ j.neubiorev.2013.10.013

Kardefelt-Winther, D., Heeren, A., Schimmenti, A., van Rooij, A., Maurage, P., \& Carras, M. (2017). How can we conceptualize behavioural addiction without pathologizing common behaviours? Addiction, 112(10), 1709-1715. doi:10.1111/add.13763

*Ko, C. H., Liu, G. C., Hsiao, S., Yen, J. Y., Yang, M. J., \& Lin, W. C. (2009). Brain activities associated with gaming urge of online gaming addiction. Journal of Psychiatric Research, 43(7), 739-747. doi:10.1016/j.jpsychires.2008.09.012

*Ko, C. H., Liu, G. C., Yen, J. Y., Chen, C. Y., Yen, C. F., \& Chen, C. S. (2011). Brain correlates of craving for online gaming under cue exposure in subjects with Internet gaming addiction and in remitted subjects. Addiction Biology, 18(3), 559-569. doi:10.1111/j.1369-1600.2011.00405.x

*Ko, C. H., Liu, G. C., Yen, J. Y., Yen, C. F., Chen, C. S., \& Lin, W. C. (2013). The brain activations for both cue-induced gaming urge and smoking craving among subjects comorbid with Internet gaming addiction and nicotine dependence. 
Journal of Psychiatric Research, 47(4), 486-493. doi:10.1016/ j.jpsychires.2012.11.008

Kochunov, P., Lancester, J., Thompson, P., Toga, A. W., Brewer, P., \& Hardies, J. (2002). An optimized individual target brain in the Talairach coordinate system. Neuroimage, 17(2), 922-927. doi:10.1006/nimg.2002.1084

*Liu, L., Yip, S. W., Zhang, J.-T., Wang, L.-J., Shen, Z.-J., \& Liu, B. (2017). Activation of the ventral and dorsal striatum during cue reactivity in Internet gaming disorder. Addiction Biology, 22(3), 791-801. doi:10.1111/adb.12338

Liu, X., Hairston, J., Schrier, M., \& Fan, J. (2011). Common and distinct networks underlying reward valence and processing stages: A meta-analysis of functional neuroimaging studies. Neuroscience and Biobehavioral Reviews, 35(5), 1219-1236. doi:10.1016/j.neubiorev.2010.12.012

*Lorenz, R. C., Krüger, J.-K., Neumann, B., Schott, B. H., Kaufmann, C., \& Heinz, A. (2012). Cue reactivity and its inhibition in pathological computer game players. Addiction Biology, 18(1), 134-146. doi:10.1111/j.1369-1600. 2012.00491.x

*McGrath, D. S., Dorbeck, A., \& Barret, S. P. (2013). The influence of acutely administered nicotine on cue-induced craving for gambling in at-risk video lottery terminal gamblers who smoke. Behavioral Pharmacology, 24(2), 124-132. doi:10.1097/FBP.0b013e32835f3cff

Meng, Y., Deng, W., Wang, H., Guo, W., Li, T., \& Lam, C. (2014). Reward pathway dysfunction in gambling disorder: A metaanalysis of functional magnetic resonance imaging studies. Behavioral Brain Research, 275, 243-251. doi:10.1016/ j.bbr.2014.08.057

Miedl, S. F., Büchel, C., \& Peters, J. (2014). Cue-induced craving increases impulsivity via changes in striatal value signals in problem gamblers. The Journal of Neuroscience, 34(13), 4750-4755. doi:10.1523/JNEUROSCI.502013.2014

Niu, G.-F., Sun, X.-J., Subrahmanyam, K., Kong, F.-C., Tian, Y., \& Zhou, Z.-K. (2016). Cue-induced craving for Internet among Internet addicts. Addictive Behaviors, 62, 1-5. doi:10.1016/j. addbeh.2016.06.012

Noori, H. R., Cosa Linan, A., \& Spanagel, R. (2016). Largely overlapping neuronal substrates of reactivity to drug, gambling, food and sexual cues: A comprehensive meta-analysis. European Neuropsychopharmacology, 26(9), 1419-1430. doi:10.1016/j.euroneuro.2016.06.013

Potenza, M. N. (2006). Should addictive disorders include non-substance-related conditions? Addiction, 101(Suppl. 1), 142-151. doi:10.1111/j.1360-0443.2006.01591.x

Potenza, M. N. (2014). Non-substance addictive behaviors in the context of DSM-5. Addictive Behaviors, 39(1), 1-2. doi:10.1016/ j.addbeh.2013.09.004

*Potenza, M. N., Steinberg, M. A., Skudlarski, P., Fulbright, R. K., Lacadie, C. M., \& Wilber, M. K. (2003). Gambling urges in pathological gambling. A functional magnetic resonance imaging study. Archives of General Psychiatry, 60(8), 828-836. doi:10.1001/archpsyc.60.8.828

Prause, N., Steele, V. R., Stalay, C., Sabatinelli, D., \& Hajcak, G. (2015). Modulation of late positive potentials by sexual images in problem users and controls inconsistent with "porn addiction”. Biological Psychology, 109, 192-199. doi:10.1016/j. biopsycho.2015.06.005
Raab, G., Elger, C. E., Neuner, M., \& Weber, B. (2011). A neurological study of compulsive buying behavior. Journal of Consumer Policy, 34(4), 401-413. doi:10.1007/s10603011-9168-3

Radua, J., \& Mataix-Cols, D. (2009). Voxel-wise meta-analysis of grey matter changes in obsessive-compulsive disorder. The British Journal of Psychiatry, 195(5), 393-402. doi:10.1192/ bjp.bp.108.055046

Radua, J., Mataix-Cols, D., Phillips, M. L., El-Hage, W., Kronhaus, D. M., \& Cardoner, N. (2012). A new metaanalytic method for neuroimaging studies that combines reported peak coordinates and statistical parametric maps. European Psychiatry, 27(8), 605-611. doi:10.1016/j.eurpsy. 2011.04.001

Robinson, T. E., \& Berridge, K. C. (1993). The neural basis of drug craving: An incentive-sensitization theory of addiction. Brain Research Reviews, 18(3), 247-291. doi:10.1016/0165-0173 (93)90013-P

Robinson, T. E., \& Berridge, K. C. (2008). The incentive sensitization theory of addiction: Some current issues. Philosophical Transactions of the Royal Society of London. Series B, Biological Sciences, 363(1507), 3137-3146. doi:10.1098/ rstb.2008.0093

Rosenthal, R. (1979). The file drawer problem and tolerance for null results. Psychological Bulletin, 86(3), 638-641. doi:10.1037/0033-2909.86.3.638

*Sharpe, L., Tarrier, N., Schotte, D., \& Spence, S. H. (1995). The role of autonomic arousal in problem gambling. Addiction, 90(11), 1529-1540. doi:10.1111/j.1360-0443.1995. tb02815.x

*Sodano, R., \& Wulfert, E. (2010). Cue reactivity in active pathological, abstinent pathological, and regular gamblers. Journal of Gambling Studies, 26(1), 53-65. doi:10.1007/ s10899-009-9146-8

*Sun, Y., Ying, H., Seetohul, R. M., Xuemei, W., Ya, Z., \& Qian, L. (2012). Brain fMRI study of crave induced by cue pictures in online game addicts (male adolescents). Behavioral Brain Research, 233(2), 563-576. doi:10.1016/j.bbr.2012.05.005

*Thalemann, R., Wölfling, K., \& Grüsser, S. M. (2007). Specific cue reactivity on computer game-related cues in excessive gamers. Behavioral Neuroscience, 121(3), 614-618. doi:10.1037/0735-7044.121.3.614

Tiffany, S., \& Wray, J. (2011). The clinical significance of drug craving. Annals of the New York Academy of Sciences, 1248(1), 1-17. doi:10.1111/j.1749-6632.2011.06298.x

*Trotzke, P., Starcke, K., Pedersen, A., \& Brand, M. (2014). Cueinduced craving in pathological buying: Empirical evidence and clinical implications. Psychosomatic Medicine, 76(9), 694-700. doi:10.1097/PSY.0000000000000126

Vevea, J. L., \& Woods, C. M. (2005). Publication bias in research synthesis: Sensitivity analysis using a priori weight functions. Psychological Methods, 10(4), 428-443. doi:10.1037/1082989X.10.4.428

von Gebsattel, V. E. (1954). Prolegolema einer medizinischen Anthropologie [Prolegolema of a medical anthropology]. Berlin, Germany: Springer.

Voon, V., Mole, T. B., Banca, P., Porter, L., Morris, L., \& Mitchell, S. (2014). Neural correlates of sexual cue reactivity in individuals with and without compulsive sexual behaviors. PLoS One, 9(7), e102419. doi:10.1371/journal.pone.0102419 
Wilson, S. J., Sayette, M. A., \& Fiez, J. A. (2004). Prefrontal responses to drug cues: A neurocognitive analysis. Nature Neuroscience, 7(3), 211-214. doi:10.1038/nn1200

*Wölfling, K., Mörsen, C. P., Duven, E., Albrecht, U., Grüsser, S. M., \& Flor, H. (2011). To gamble or not to gamble: At risk for craving and relapse - Learned motivated attention in pathological gambling. Biological Psychology, 87(2), 275-281. doi:10.1016/j.biopsycho.2011.03.010

Wrase, J., Grüsser, S. M., \& Heinz, A. (2006). Cue-induced alcohol craving. Neurobiological correlates and clinical relevance. Der Nervenarzt, 77(9), 1051-1063. doi:10.1007/ s00115-006-2067-1 